\title{
A Low-Complexity Precoding Scheme for the Downlink of Multi-cell Multi-user MIMO AF System
}

\author{
Yusuf Sambo, Fabien Héliot, and Muhammad Ali Imran \\ Centre for Communication Systems Research, University of Surrey, \\ Guildford GU2 7HX, UK, Email: yusambo@ieee.org
}

\begin{abstract}
Because of its simplicity, amplify-and-forward (AF) is one of the most popular cooperative relaying technique. Relays are used in cooperative communication to improve reliability, coverage or spectral efficiency of cell-edge users. However, relays tend to increase the interferences seen by users of adjacent cells, particularly by the cell-edge users, when used in multi-cell systems. In this paper, we propose a low-complexity precoding scheme to mitigate the effect of other-cell interference (OCI) in cooperative communication. The scheme is designed by taking into account the interference plus noise covariance matrix of each user for mitigating the interference at each receiver by means of precoding at the relay node. Simulation results show the effectiveness of the proposed scheme, both in terms of sum-rate and computational complexity, when compared to other existing OCI-aware precoding algorithms for AF.
\end{abstract}

Index Terms-Amplify-and-forward, MIMO systems, multicell, downlink, precoding

\section{INTRODUCTION}

In wireless communication, cooperative techniques such as decode-and-forward (DF) and amplify-and-forward (AF) have in recent years attracted a lot of research interests [1]-[3]. $\mathrm{AF}$ is a nonregenerative technique in which a source node (SN) broadcasts its signal to a relay node ( $\mathrm{RN})$ as well as a destination node (DN). The RN is utilized for amplifying and forwarding this signal to the DN in order to improve the reliability and/or the spectral efficiency of the system.

In the early work on multi-input multi-output (MIMO) AF and relaying communication [3], the RN was utilized as a dumb equal gain amplifier, and later in the watershed works of [4] and [5], it has been demonstrated that the RN can also perform precoding in order to improve the spectral efficiency of the single cell single user MIMO system. Then, numerous works have looked at extending this concept to various other scenarios such as single cell multi-carrier MIMO AF [6], single cell multi-user (MU)-MIMO AF [7], [8] or multi-cell MU-MIMO AF [9].

The concept of precoding itself derives from single cell MUMIMO communication, where various theoretical and practical schemes such as [10] and [11], [12] have been developed over the years for improving the spectral efficiency by mitigating intra-cell interference in a single cell context. In parallel to the development of precoding techniques for single cell MIMOAF and MU-MIMO AF, multi-cell MU-MIMO precoding has recently been investigated in [13] and an efficient precoder based on the channel block diagonalization (CBD) scheme of [11] has been proposed. As pointed out in [14], interference that is produced by neighboring cells significantly degrades the cell-edge user and overall sum-rate performances in multicell system. Thus, the other-cell interference (OCI)-aware precoding scheme of [13] provides a practical solution to this problem but for point-to-point multi-cell communication. In cooperative multi-cell communication, we expect this problem to be exacerbated since one of the induced effects of relaying will be to increase the OCI due to neighboring cell-edge users. As a matter of fact, we have proposed in [9] two precoding schemes for tackling this problem, namely the AF-enhanced CBD (ECBD) and AF-enhanced constrained gradient search for DL (ECGSDL) schemes. The latter performs better than the former in terms of spectral efficiency but is more demanding in terms of computational complexity.

In this paper, we propose a novel precoding method for the multi-cell MU-MIMO AF scenario based on the regularized channel inversion (RCI) approach of [12], which was proposed for the single cell MU-MIMO scenario, by modifying it for the MU-MIMO AF case and integrating OCI effects in its precoding structure at the RN. The resulting scheme combines the advantages of our two previous schemes in [9], i.e. it provides similar sum-rate performance as the ECGSDL scheme and with the same order of computational complexity than the ECBD scheme. In Section II, we describe the system model for the DL of multi-cell MU MIMO AF and formulate the sum-rate equation in this scenario. Using this equation as an objective function, we design our novel multi-cell MU-MIMO AF precoding scheme in Section III by considering as in [9] that the SN-RN and RN-DN link channel state information (CSI) and the interference plus noise covariance matrix of each cell-edge user are known at the RN. In Section IV, we then present the sum-rate and computational complexity performances of our novel OCI-aware scheme for various settings. These results show that our new algorithm outperforms the two previous existing OCI-aware algorithms for AF. Finally, Section V concludes the paper.

\section{Multi-Cell MU-MiMO AF System Description}

We consider the downlink of the MU-MIMO AF system in presence of OCI as in Fig. 1, where the direct link is weak in comparison with the relay link and transmission occurs in 


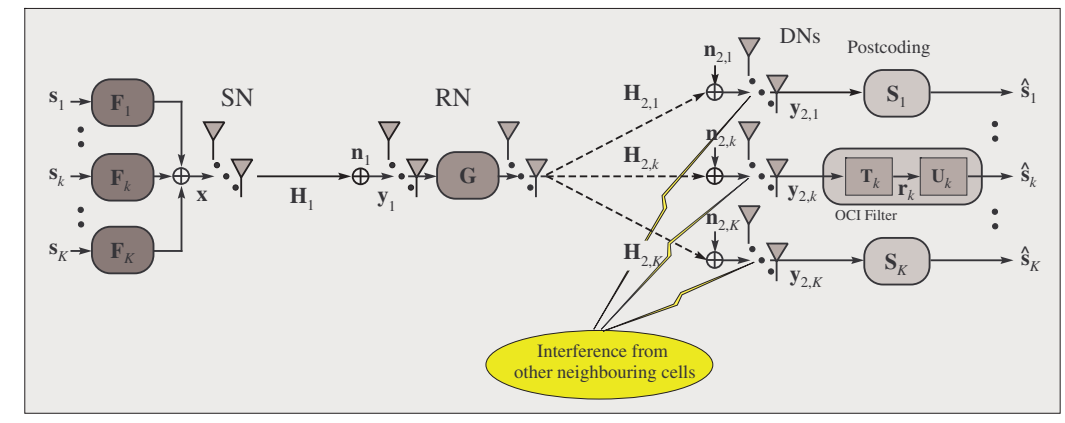

Fig. 1. DL of MU-MIMO AF system in presence of OCI.

two phases of equal duration as in [5]. In the first phase, a SN with $n$ antennas broadcasts its signal $\mathbf{x}$ over a MIMO channel $\mathbf{H}_{1} \in \mathbb{C}^{q \times n}$ to a nonregenerative RN with $q$ antennas, which is received as

$$
\mathbf{y}_{1}=\mathbf{H}_{1} \mathbf{x}+\mathbf{n}_{1}
$$

In the second phase the signal $\mathbf{y}_{1}$ is amplified at the $\mathrm{RN}$ by means of the precoding matrix $\mathbf{G} \in \mathbb{C}^{q \times q}$ and is then transmitted over a MIMO channel $\mathbf{H}_{2, k} \in \mathbb{C}^{r_{k} \times q}$ to each of the $K$ DNs with $r_{k}$ antennas. At each DN, the received signal $\mathbf{y}_{2, k}$ is corrupted by noise $\mathbf{n}_{2, k}$ as well as OCI $\mathbf{n}_{\mathrm{OCI}, k}$ such that

$$
\mathbf{y}_{2, k}=\mathbf{H}_{2, k} \mathbf{G H}_{1} \mathbf{x}+\mathbf{H}_{2, k} \mathbf{G n}_{1}+\mathbf{n}_{2, k}+\mathbf{n}_{\mathrm{OCI}, k} .
$$

Note that $\mathbf{n}_{1} \in \mathbb{C}^{q \times 1}$ and $\mathbf{n}_{2, k} \in \mathbb{C}^{r_{k} \times 1}$ are vectors of independent zero-mean complex Gaussian noise entries with a variance of $\sigma_{1}^{2}$ and $\sigma_{2, k}^{2}$, respectively. The signal $\mathbf{y}_{2, k}$ is then post-processed by using $\mathbf{S}_{k}=\mathbf{U}_{k} \mathbf{T}_{k}$, where $\mathbf{T}_{k} \in \mathbb{C}^{r_{k} \times r_{k}}$ is the $k$-th user OCI mitigation filter and $\mathbf{U}_{k} \in \mathbb{C}^{n \times r_{k}}$, such that the $k$-th estimated transmit message $\widehat{\mathbf{s}}_{k}$ is given by $\widehat{\mathbf{s}}_{k}=\mathbf{S}_{k} \mathbf{y}_{2, k}$. In addition, the $k$-th DN received signal after OCI mitigation can be expressed as

$$
\mathbf{r}_{k}=\mathbf{H}_{k} \mathbf{G H}_{1} \mathbf{x}+\mathbf{H}_{k} \mathbf{G n}_{1}+\mathbf{T}_{k}\left(\mathbf{n}_{2, k}+\mathbf{n}_{\mathrm{OCI}, k}\right),
$$

where $\mathbf{H}_{k}=\mathbf{T}_{k} \mathbf{H}_{2, k}$.

According to Fig. 1, we define the $\mathrm{SN}$ transmit signal as $\mathbf{x}=$ $\sum_{k=1}^{K} \mathbf{F}_{k} \mathbf{s}_{k}$, where $\mathbf{F}_{k} \in \mathbb{C}^{n \times n}$ is the $k$-th user SN precoder and $\mathbf{s}_{k}=\left[\begin{array}{lll}\mathbf{0}^{1 \times \alpha_{k}} & \overline{\mathbf{s}}_{k}^{\dagger} & \mathbf{0}^{1 \times\left(n-\alpha_{k+1}\right)}\end{array}\right]^{\dagger}$, with $\overline{\mathbf{s}}_{k} \in \mathbb{C}^{l_{k} \times 1}$ being the $k$-th message of length $l_{k}, 0^{1 \times \alpha_{k}}$ being an all zero vector of length $\alpha_{k}, \alpha_{k}=\sum_{j=1}^{k-1} l_{j}$ and $\alpha_{K+1}=\sum_{k=1}^{K} l_{k}=l$. Thus, $\mathrm{E}\left\{\mathbf{s}_{k} \mathbf{s}_{k}^{\dagger}\right\}=\mathbf{I}_{l_{k}}$, where $\mathbf{I}_{l_{k}}$ is a $l_{k} \times l_{k}$ identity matrix, $\mathrm{E}\{$.$\} stands for the expectation, \{.\}^{\dagger}$ is the conjugate transpose operator, and we assume that $l_{k} \leq r_{k}$ and $l \leq n$. As far as the OCI model is concerned, we utilize the model of [13], where $\mathbf{n}_{\mathrm{OCI}, k}=\mathbf{H}_{\mathrm{OCI}, k} \mathbf{x}_{\mathrm{OCI}, k}$ with $\mathbf{H}_{\mathrm{OCI}, k} \in \mathbb{C}^{r_{k} \times n_{\mathrm{OCl}, k}}$ and $\mathbf{x}_{\mathrm{OCI}, k} \in \mathbb{C}^{n_{\mathrm{OCI}, k} \times 1}$ being the MIMO OCI channel and OCI signal with $n_{\mathrm{OCI}, k}$ co-channel interferers from the neighboring cells, respectively. Using (3) and the previous assumptions, the mutual information (MI) of each user is given by [15]

$$
\begin{aligned}
I\left(\mathbf{r}_{k} ; \mathbf{s}_{k}\right)= & \frac{1}{2} \log _{2} \mid \mathbf{I}_{r_{k}}+\mathbf{H}_{k} \mathbf{G}\left(\mathbf{R}_{\mathbf{y}_{1}}-\mathbf{R}_{\mathbf{n}_{1}, k}\right) \mathbf{G}^{\dagger} \mathbf{H}_{k}^{\dagger} \\
& \times\left(\mathbf{H}_{k} \mathbf{G R}_{\mathbf{n}_{1}, k} \mathbf{G}^{\dagger} \mathbf{H}_{k}^{\dagger}+\mathbf{T}_{k} \mathbf{R}_{\mathbf{n}_{\mathrm{OCl}}, k} \mathbf{T}_{k}^{\dagger}\right)^{-1} \mid
\end{aligned}
$$

where the factor $1 / 2$ stands for the dual-phase transmission, $\mathbf{R}_{\mathbf{y}_{1}}=\mathrm{E}\left\{\mathbf{y}_{1} \mathbf{y}_{1}^{\dagger}\right\}=\sigma_{1}^{2} \mathbf{I}_{q}+\mathbf{H}_{1} \mathbf{R}_{\mathbf{x}} \mathbf{H}_{1}^{\dagger}$ is the transmit covariance matrix, $\mathbf{R}_{\mathbf{n}_{1}, k}=\sigma_{1}^{2} \mathbf{I}_{q}+\mathbf{H}_{1}\left(\mathbf{R}_{\mathbf{x}}-\mathbf{R}_{k} \mathbf{R}_{k}^{\dagger}\right) \mathbf{H}_{1}^{\dagger}$ is the $k$-th noise plus intra-cell interference covariance matrix and $\mathbf{R}_{\mathbf{x}}=\sum_{k=1}^{K} \mathbf{F}_{k} \mathbf{F}_{k}^{\dagger}$. Moreover, $\mathbf{R}_{\mathbf{n}_{\mathrm{OCl}}, k}=\sigma_{2, k}^{2} \mathbf{I}_{r_{k}}+$ $\mathbf{H}_{\mathrm{OCI}, k} \mathbf{R}_{\mathrm{OCI}, k} \mathbf{H}_{\mathrm{OCI}, k}^{\dagger}$ is the noise plus OCI covariance matrix for the $k$-th $\mathrm{DN}$ and $\mathbf{R}_{\mathrm{OCI}, k}=\mathrm{E}\left\{\mathbf{x}_{\mathrm{OCI}, k} \mathbf{x}_{\mathrm{OCI}, k}^{\dagger}\right\}$. Using (4), the relay link sum-MI, or equivalently sum-rate, can be expressed as

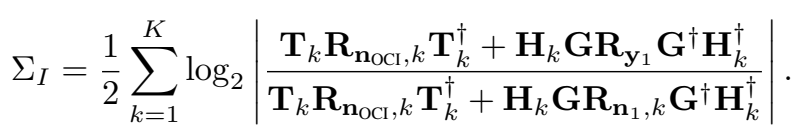

Additionally, the $k$-th DN $\mathbf{U}_{k}$ matrix can be obtained as $\mathbf{U}_{k}=$

$$
\mathbf{F}_{k}^{\dagger}\left(\mathbf{H}_{k} \mathbf{G H}_{1}\right)^{\dagger}\left(\mathbf{H}_{k} \mathbf{G} \mathbf{H}_{1} \mathbf{R}_{\mathbf{x}}\left(\mathbf{H}_{k} \mathbf{G} \mathbf{H}_{1}\right)^{\dagger}+\mathbf{T}_{k} \mathbf{R}_{\mathbf{n}_{\mathrm{OCl}}, k} \mathbf{T}_{k}^{\dagger}\right)^{-1}
$$

by solving the gradient of $\mathrm{E}\left\{\left(\widehat{\mathbf{s}}_{k}-\mathbf{s}_{k}\right)\left(\widehat{\mathbf{s}}_{k}-\mathbf{s}_{k}\right)^{\dagger}\right\}=0$, when each DN $k$ uses a linear Minimum Mean Squared Error (MMSE) receiver [9].

\section{Regularized ChanNel INVERsion PRECODING FOR OCI Mitigation In Multi-CEll MU-MiMO AF}

In this section, we propose an enhanced version of the regularized channel inversion algorithm in [12] to mitigate the effect of OCI and maximize the sum-rate of multi-cell MUMIMO AF system based on the assumption that the transmitter has knowledge of the interference plus noise covariance matrix for each DN. Unlike full cooperation, this approach is quite practical because each DN can easily estimate its covariance matrix without the need for synchronization, training sequence or additional pilot symbols. We also assume as in [9] that the RN has knowledge of the CSI of the SN-RN and all the RNDN links, i.e. $\mathbf{H}_{1}$ and $\mathbf{H}_{2, k}$, respectively, and that the $\mathrm{SN}$ has knowledge of $\mathbf{H}_{1}$ where $\mathbf{H}_{1}=\mathbf{U} \widehat{\boldsymbol{\Lambda}}^{\frac{1}{2}} \mathbf{V}^{\dagger}$ by using singular value decomposition (SVD). Note that $\mathbf{U} \in \mathbb{C}^{q \times q}$ as well as 
$\mathbf{V} \in \mathbb{C}^{n \times n}$ are unitary matrices, and $\widehat{\Lambda}$ is a $q \times n$ rectangular diagonal matrix. In addition, $\boldsymbol{\Lambda}=\widehat{\Lambda}^{\frac{1}{2}} \widehat{\Lambda}^{\frac{1}{2} \dagger}$ is a $q \times q$ diagonal matrix with diagonal elements $\lambda_{i} \in \mathbb{R}_{+}$. Assuming that the SN has the knowledge of $\mathbf{V}$, we define $\mathbf{F}_{k}$ as

$$
\mathbf{F}_{k}=\widehat{\mathbf{F}}_{k} \mathbf{V}
$$

where $\widehat{\mathbf{F}}_{k}=\operatorname{diag}\left(\sqrt{\mathbf{p}_{k}}\right)$ is a $n \times n$ diagonal matrices and $\mathbf{p}_{k}=\left[\begin{array}{lll}\mathbf{0}^{1 \times \alpha_{k}} & \left\{p_{\alpha_{k}+1}, \ldots, p_{\alpha_{k}+l_{k}}\right\} & \mathbf{0}^{1 \times\left(n-\alpha_{k+1}\right)}\end{array}\right]$.

By definition, the matrix $\mathbf{R}_{\mathbf{n}_{\mathrm{OCI}}, k}$ is Hermitian positive definite and, hence, is equivalent to $\mathbf{R}_{\mathbf{n}_{\mathrm{OCI}}, k}=\mathbf{D}_{k} \mathbf{D}_{k}^{\dagger}$ when using Cholesky decomposition. Using this result, we define $\mathbf{T}_{k}=\sigma_{2, k} \mathbf{D}_{k}^{-1}$ and insert it in (5) such that

$$
\Sigma_{I}=\frac{1}{2} \sum_{k=1}^{K} \log _{2}\left|\frac{\sigma_{2, k}^{2} \mathbf{I}_{r_{k}}+\mathbf{H}_{k} \mathbf{G} \mathbf{R}_{\mathbf{y}_{1}} \mathbf{G}^{\dagger} \mathbf{H}_{k}^{\dagger}}{\sigma_{2, k}^{2} \mathbf{I}_{r_{k}}+\mathbf{H}_{k} \mathbf{G} \mathbf{R}_{\mathbf{n}_{1}, k} \mathbf{G}^{\dagger} \mathbf{H}_{k}^{\dagger}}\right| .
$$

It can be noticed that (7) is similar to the single cell relay link sum-rate formulation, which is given in equation (5) of [7], but where $\mathbf{H}_{2, k}$ has been replaced by $\mathbf{H}_{k}$. In effect, by utilizing $\mathbf{T}_{k}=\sigma_{2, k} \mathbf{D}_{k}^{-1}$ as our OCI mitigation filter, the OCI has been compounded with $\mathbf{H}_{2, k}$ to form the equivalent channel $\mathbf{H}_{k}$. As a result, each DN needs to feedback $\mathbf{H}_{k}$ when OCI is strong as opposed to $\mathbf{H}_{2, k}$ when OCI is negligible.

As in [12] for the MIMO scenario, our precoder $\mathbf{G}$ at the $\mathrm{RN}$ for the multi-cell MU-MIMO AF case is designed in two stages. In the first stage, we design the precoder to cancel intracell interference using regularized channel inversion. Then, optimal power allocation is performed in the second stage. We express our RN precoder structure as

$$
\mathbf{G}=\mathbf{W} \widehat{\mathbf{G}} \mathbf{U}^{\dagger}
$$

where $\mathbf{W}=\left[\mathbf{W}_{1}, \mathbf{W}_{2}, \ldots, \mathbf{W}_{K}\right], \mathbf{W}_{k} \in \mathbb{C}^{q \times l_{k}}$, and $\widehat{\mathbf{G}}=$ $\operatorname{diag}(\sqrt{\mathbf{g}}), \mathbf{g}=\left\{g_{1}, \ldots, g_{q}\right\}$. While multi-cell interference, i.e. OCI, is mitigated by using $\mathbf{T}_{k}$, intra-cell interference is mitigated by utilizing $\mathbf{W}_{k}$ and $\widehat{\mathbf{G}}$ enables power allocation at the RN. With the RN having perfect knowledge of $\mathbf{H}_{k}, \mathbf{W}_{k}$ can be chosen to project $\mathbf{x}_{k}=\mathbf{F}_{k} \mathbf{s}_{k}$ towards the null space of $\left\{\mathbf{H}_{j}\right\}_{j \neq k}$ such that intra-cell interference is suppressed at all other $K-1 \mathrm{DNs}$ and at the same time the mean squared error (MSE) is minimized. We express the regularized channel inversion matrix as

$$
\mathbf{Q}_{k, i}=\left(\mathbf{H}_{k, i}^{\dagger} \mathbf{H}_{k, i}+\eta \mathbf{I}_{q}\right)^{-1} \mathbf{H}_{k, i}^{\dagger},
$$

where $\mathbf{H}_{k, i}=\left[\mathbf{h}_{k, i} \overline{\mathbf{H}}_{k}^{T}\right]^{T}, \overline{\mathbf{H}}_{k}=\left[\mathbf{H}_{1}^{T} \ldots \mathbf{H}_{k-1}^{T} \mathbf{H}_{k+1}^{T} \ldots \mathbf{H}_{K}^{T}\right]^{T}$, $\{.\}^{T}$ denotes the transpose operator, and the optimal $\eta$, given as $\eta=l / \gamma_{2}$, maximizes the signal-to-interference-plus-noise ratio. Moreover, $\mathbf{h}_{k, i}$ represents the $i$-th column of $\mathbf{H}_{k}^{T}$ and $\gamma_{2, k}=P_{2} / \sigma_{2}^{2}$ is the average transmit SNR of the RN-DN link when assuming that $\sigma_{2, k}^{2}=\sigma_{2}^{2}$ for $k=1, \ldots, K$ and where $P_{2}$ is the total transmit power at the RN.

Representing $\mathbf{w}_{k, i}^{0}$ as the first column vector of $\mathbf{Q}_{k, i}, \mathbf{w}_{k, i}^{0}$ is obtained from (9) as

$$
\mathbf{w}_{k, i}^{0}=\left(\sum_{\substack{j=1 \\ j \neq k}}^{K} \mathbf{H}_{j}^{\dagger} \mathbf{H}_{j}+\left(\mathbf{h}_{k, i}^{T}\right)^{\dagger} \mathbf{h}_{k, i}^{T}+\eta \mathbf{I}_{q}\right)^{-1}\left(\mathbf{h}_{k, i}^{T}\right)^{\dagger}
$$

for $i=1, \ldots, l_{k}$. The projection vector $\mathbf{w}_{k, i}^{0}$ for each data stream is found independently between streams $i=1, \ldots, l_{k}$ of the same user $k$. Let us define $\mathbf{H}_{k}^{e}$ as the RN-DN link effective channel matrix for the $k$-th $\mathrm{DN}$ such that $\mathbf{H}_{k}^{e}=\mathbf{H}_{k} \mathbf{M}_{k}$ and $\mathbf{W}_{k}$ as $\mathbf{W}_{k}=\mathbf{M}_{k} \mathbf{V}_{k}^{e}$, where $\mathbf{V}_{k}^{e}$ represents the right singular matrix of $\mathbf{H}_{k}^{e}$ obtained via SVD, which diagonalizes $\mathbf{H}_{k}^{e}$. The columns of $\mathbf{M}_{k}$ are constrained to lie within the subspace of the vectors $\left\{\mathbf{w}_{k, 1}^{0}, \mathbf{w}_{k, 2}^{0}, \ldots, \mathbf{w}_{k, l_{k}}^{0}\right\}$. Denoting $\mathbf{m}_{k, i}$ as the $i$-th column of $\mathbf{M}_{k}$, vectors $\left\{\mathbf{m}_{k, 1}, \mathbf{m}_{k, 2}, \ldots, \mathbf{m}_{k, l_{k}}\right\}$ are found as an arbitrary orthonormal basis of the vector space of vectors $\left\{\mathbf{w}_{k, 1}^{0}, \mathbf{w}_{k, 2}^{0}, \ldots, \mathbf{w}_{k, l_{k}}^{0}\right\}$ such that

$$
\mathbf{m}_{k, i} \in R\left(\left[\mathbf{w}_{k, 1}^{0}, \mathbf{w}_{k, 2}^{0}, \ldots, \mathbf{w}_{k, l_{k}}^{0}\right]\right),
$$

where $R($.$) represents the column space of a matrix. Note$ that Gram-Schmidt orthogonalization can be used to obtain the columns of $\mathbf{M}_{k}$.

By inserting (6) and (8) into (7), the aggregate mutual information of the relay can be re-expressed as

$$
\Sigma_{I}(\mathbf{g})=\frac{1}{2} \sum_{k=1}^{K} \prod_{i=1}^{l_{k}} \log _{2}\left(\frac{\sigma_{2}^{2}+g_{u} \omega_{u}\left(\sigma_{1}^{2}+\lambda_{u} p_{u}\right)}{\sigma_{2}^{2}+g_{u} \omega_{u}}\right),
$$

where $u=\alpha_{k}+i$ and $\omega_{u}$ is the $i$-th nonnegative eigenvalue of $\mathbf{H}_{k} \mathbf{M}_{k} \mathbf{M}_{k}^{\dagger} \mathbf{H}_{k}^{\dagger}$, which are sorted in descending order for each user. The optimal power allocation is then obtained by solving the following concave optimization problem

$$
\begin{aligned}
& \max _{\mathbf{g}} \Sigma_{I}(\mathbf{g}) \\
& \text { s.t. } g_{u} \geq 0 ; \sum_{k=1}^{K} \sum_{i=1}^{l_{k}} g_{u}\left(\sigma_{1}^{2}+\lambda_{u} p_{u}\right) \leq P_{2}
\end{aligned}
$$

Using Lagrange method, the following optimum solution is obtained

$$
\begin{aligned}
g_{u} & =\frac{1}{2 \omega_{u}\left(\sigma_{1}^{2}+p_{u} \lambda_{u}\right)}\left[-\left(\sigma_{1}^{2}+p_{u} \lambda_{u}+1\right) \sigma_{2, k}^{2}\right. \\
& \left.+\sigma_{2, k}^{2} \sqrt{4 \mu \omega_{u}\left(\sigma_{1}^{2}+p_{u} \lambda_{u}-1\right)+\left(\sigma_{1}^{2}+p_{u} \lambda_{u}-1\right)^{2} \sigma_{2, k}^{2}}\right]_{+}
\end{aligned}
$$

where $[n]_{+}=\max \{0, n\}$ and $\mu \geq 0$ is the Lagrange multiplier. The starting point for the search of the $\mu$ that optimizes (14) is given by

$\mu_{\min }=\max _{u}\left\{\frac{\left(\sigma_{1}^{2}+p_{u} \lambda_{u}+1\right)^{2}-\left(\sigma_{1}^{2}+p_{u} \lambda_{u}-1\right)^{2} \sigma_{2, k}^{2}}{4 \mu \omega_{u}\left(\sigma_{1}^{2}+p_{u} \lambda_{u}-1\right)}\right\}$.

The Newton-Raphson method can be used to update $\mu$ until the optimal $\mu$, i.e. $\mu^{\star}$ is found. Let

$$
f(\mu)=\sum_{k=1}^{K} \sum_{i=1}^{l_{k}} g_{u}\left(\sigma_{1}^{2}+\lambda_{u} p_{u}\right)-P_{2},
$$

where $g_{u}$ is obtained from (14) and $\mu^{\star}$ is chosen to fulfil $f(\mu)<\epsilon$, with $\epsilon \ll 1$. 


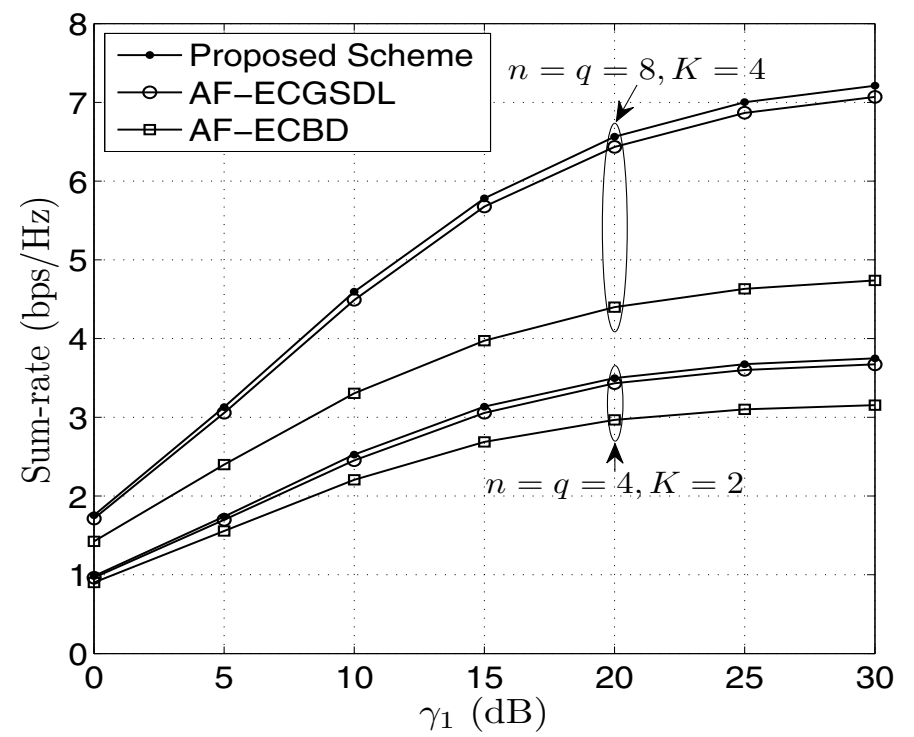

Fig. 2. Sum-rate performance of our novel OCI-aware scheme in presence of OCI against the precoding schemes of [9] for $n=q=4, K=2$ and $n=q=8, K=4$ when $\gamma_{2, k}=10 \mathrm{~dB}, \gamma_{\mathrm{OCI}, k}=20 \mathrm{~dB}, r_{k}=l_{k}=2$, and $n_{\mathrm{OCI}, k}=1$.

\section{REsults}

In this section, we compare simulation results of our proposed algorithm against the AF-ECBD and AF-ECGSDL schemes of [9] both in terms of sum-rate and computational complexity.

In our simulations, $\gamma_{1}=P_{1} / \sigma_{1}^{2}, \gamma_{2, k}=P_{2} / \sigma_{2, k}^{2}$ and $\gamma_{\mathrm{OCI}, k}=P_{\mathrm{OCI}, k} / \sigma_{2, k}^{2}$ denote the SN-RN link SNR, the $k$ th RN-DN link SNR and the $k$-th DN's interference-to-noise ratio, respectively, where $P_{1}$ is the average transmit power of the $\mathrm{SN}$ and $P_{\mathrm{OCI}, k}$ is the average power of each interference signal. Moreover, we consider that $\sigma_{1}^{2}=\sigma_{2, k}^{2}=1$, for $k=1, \ldots, K$. In order to assess the capacity degradation due to OCI in the worst case scenario, we assume as in [13] that $\mathbf{R}_{\mathrm{OCI}, k}=\left(P_{\mathrm{OCI}, k} / n_{\mathrm{OCI}, k}\right) \mathbf{I}_{n_{\mathrm{OCI}, k}}$. We also assume equal power allocation at the SN such that $p_{\alpha_{k}+j}=P_{1} / l$, for $j=1, \ldots, l_{k}$ and $k=1, \ldots, K$ and set $\epsilon=10^{-5}$. Furthermore, we consider the same sorting of the entries of $\boldsymbol{\Lambda}$ as in [9]. For instance if $K=4, q=8$ and $l_{k}=2, k=1, \ldots, K$, then $\lambda_{1}, \lambda_{2}$, $\lambda_{3}$ and $\lambda_{7}$ would be the first, fifth, second and fourth largest eigenvalues of $\Lambda$, respectively.

Figure 2 shows the sum-rate performance comparison of the proposed scheme and the AF-ECBD and AF-ECGSDL algorithms in [9], with respect to the SN-RN link SNR, $\gamma_{1}$, in $\mathrm{dB}$. The comparison is made for $n=q=8, K=4$ and $n=q=4, K=2$. In this figure, we set $\gamma_{2, k}=10$ $\mathrm{dB}, \gamma_{\mathrm{OCI}, k}=20 \mathrm{~dB}, r_{k}=l_{k}=2$ and $n_{\mathrm{OCI}, k}=1$, for $k=1, \ldots, K$. It can be seen from the results that our proposed scheme outperforms the AF-ECBD algorithm and is slightly better than the AF-ECGSDL algorithm and the performance gap between the proposed scheme and the AF-ECBD and AF-ECGSDL algorithms increases as the size of the MIMO system increases. For example, our proposed scheme provides a performance gain of about $2.4 \mathrm{bps} / \mathrm{Hz}$ and $0.6 \mathrm{bps} / \mathrm{Hz}$ over

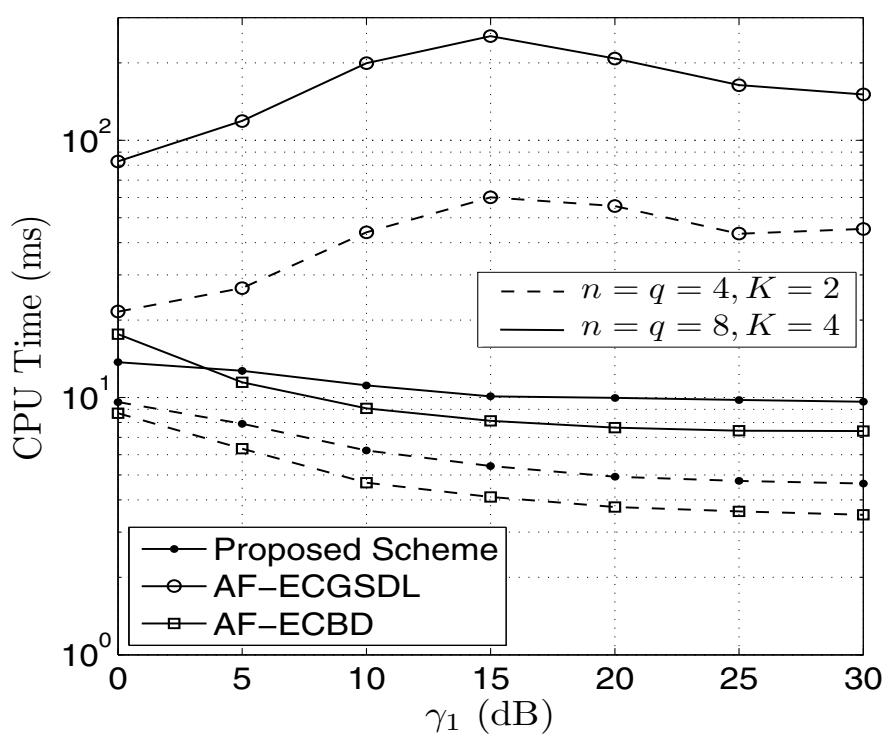

Fig. 3. Computational complexity of our novel OCI-aware scheme in presence of OCI against the precoding schemes of [9] for the same settings as in Fig. 2.
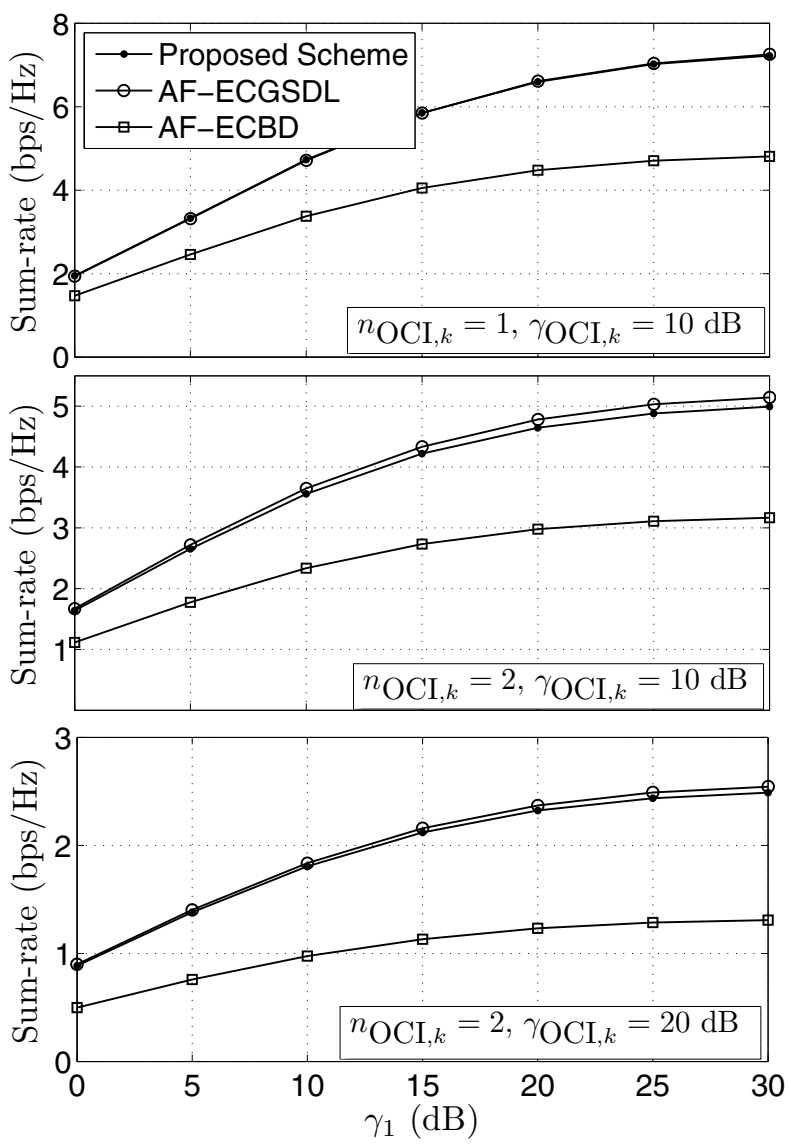

Fig. 4. Sum-rate performances of our novel OCI-aware scheme in presence of OCI against the precoding schemes of [9] for $n=q=8, K=4$ when $\gamma_{2, k}=10 \mathrm{~dB}, r_{k}=l_{k}=2$, and various OCI scenarios. 
AF-ECBD for $K=2$ and 4, respectively, at high SNR. Also, there is a performance gain of about $0.6 \mathrm{bps} / \mathrm{Hz}$ and $0.1 \mathrm{bps} / \mathrm{Hz}$ over the AF-ECGSDL algorithm for $K=4$ and $K=2$, respectively.

In Fig. 3, we compare the proposed scheme against the AF-ECBD and AF-ECGSDL algorithms in terms of computational complexity. The comparison was made for the same configuration as in Fig. 2. We use the mean CPU execution time of the algorithms in milliseconds (ms) as a metric for the comparison. The results show that our proposed scheme has about the same computational complexity as the AF-ECBD algorithm, but is far less computationally demanding than the AF-ECGSDL algorithm. Also, the computational complexity of the algorithms increases as the size of the MIMO system increases. It can be seen that our novel algorithm is at least 8 and 10 times less computationally demanding than the AF-ECGSDL algorithm for $K=2$ and 4, respectively. Meanwhile, it is only about 1.3 times more computationally demanding than the AF-ECBD algorithm for both $K=2$ and 4.

In Fig. 4, we compare the sum-rate performance of our algorithm against the AF-ECBD and AF-ECGSDL algorithms in [9] for various values of $n_{\mathrm{OCI}, k}$ and $\gamma_{\mathrm{OCI}, k}$ when $n=q=8$, $K=4, r_{k}=l_{k}=2$ and $\gamma_{2, k}=10 \mathrm{~dB}$, for $k=1, \ldots, K$. Figures 4 (a), (b) as well as (c) depict the sum-rate performance comparison for $n_{\mathrm{OCI}, k}=1$ and $\gamma_{\mathrm{OCI}, k}=10 \mathrm{~dB}$, $n_{\mathrm{OCI}, k}=2$ and $\gamma_{\mathrm{OCI}, k}=10 \mathrm{~dB}$ as well as $n_{\mathrm{OCI}, k}=2$ and $\gamma_{\mathrm{OCI}, k}=20 \mathrm{~dB}$, respectively. It can be seen from Fig. 4 that our proposed scheme exhibits about the same performance as the AF-ECGSDL algorithm and has a higher sum-rate performance than the AF-ECBD for any values of $n_{\mathrm{OCI}, k}$ and $\gamma_{\mathrm{OCI}, k}$. It can also be observed that sum-rate performance decreases as the number of interfering sources increases from $n_{\mathrm{OCI}, k}=1$ to $n_{\mathrm{OCI}, k}=2$ because they affect both streams of each user compared to when only one stream per user is affected, as in the case of $n_{\mathrm{OCI}, k}=1$. Obviously, performances are also degraded when the power of the interfering sources increases.

\section{CONCLUSION}

In this paper, we have proposed a low complexity precoding algorithm for the DL of multi-cell MU-MIMO AF communication based on the regularized channel inversion approach that takes advantage of the knowledge about the OCI covariance matrix of each user to mitigate $\mathrm{OCI}$ and maximize the sum-rate performance of the system. The proposed scheme is practical as it does not require multi-cell cooperation or dirty paper coding for mitigating both the effects of OCI and intra- cell interference. Simulation results indicate that our proposed scheme outperforms the low complexity AF-ECBD algorithm in [9] but for the same order of complexity and provides similar performance as the high complexity AF-ECGSDL algorithm in [9] but with reduced complexity.

\section{ACKNOWLEDGMENT}

The research leading to these results has received funding from the European Commission's Seventh Framework Programme FP7/2007-2013 under grant agreement $n^{\circ}$-223937 project SMART-Net.

\section{REFERENCES}

[1] A. Sendonaris, E. Erkip, and B. Aazhang, "User Cooperation Diversity Part I \& II- System Description / Implementation Aspects and Performance Analysis," IEEE Trans. Commun., vol. 51, no. 11, pp. 1927-1948, Nov. 2003.

[2] A. Nosratinia, T. E. Hunter, and A. Hedayat, "Cooperative Communication in Wireless Networks," IEEE Commun. Mag., vol. 42, no. 10, pp. 74-80, Oct. 2004

[3] J. N. Laneman, D. N. C. Tse, and G. W. Wornell, "Cooperative Diversity in Wireless Networks: Efficient Protocols and Outage Behavior," IEEE Trans. Inf. Theory, vol. 50, no. 12, pp. 3062-3080, Dec. 2004.

[4] Y. Fan and J. Thompson, "MIMO Configurations for Relay Channels: Theory and Practice," IEEE Trans. Wireless Commun., vol. 6, no. 5, pp. 1774-1786, May 2007.

[5] O. Muñoz-Medina, J. Vidal, and A. Agustín, "Linear Transceiver Design in Nonregenerative Relays with Channel State Information," IEEE Trans. Signal Process., vol. 55, no. 6, pp. 2593-2604, Jun. 2007.

[6] I. Hammerström and A. Wittneben, "Power Allocation Schemes for Amplify-and-Forward MIMO-OFDM Relay Links," IEEE Trans. Wireless Commun., vol. 6, no. 8, pp. 2798-2802, Aug. 2007.

[7] F. Héliot, R. Hoshyar, and R. Tafazolli, "Power Allocation for the Downlink of Nonregenerative Cooperative Multi-User MIMO Communication system," in Proc. IEEE PIMRC, Istanbul, Turkey, Sep. 2010.

[8] Y. Yu and Y. Hua, "Power Allocation for a MIMO Relay System with Multiple-Antenna Users," IEEE Trans. Signal Process., vol. 58, no. 5 , pp. 2823-2835, May 2010.

[9] F. Héliot, U. Asif, R. Hoshyar, and R. Tafazolli, "Other-Cell Interference Aware Precoding for the Downlink of Multi-User MIMO AF Communication," in Proc. IEEE VTC-Spring, Budapest, Hungary, May 2011.

[10] N. Jindal, W. Rhee, S. Vishwanath, S. A. Jafar, and A. Goldsmith, "Sum Power Iterative Water-Filling for Multi-Antenna Gaussian Broadcast Channels," IEEE Trans. Inf. Theory, vol. 51, no. 4, pp. 1570-1580, Apr. 2005.

[11] Q. H. Spencer, A. L. Swindlehurst, and M. Haardt, "Zero-Forcing Methods for Downlink Spatial Multiplexing in Multiuser MIMO Channels," IEEE Trans. Signal Process., vol. 52, no. 2, pp. 461-471, Feb. 2004.

[12] H. Lee, K. Lee, B. M. Hochwald, and I. Lee, "Regularized Channel Inversion for Multiple-Antenna Users in Multiuser MIMO Downlink," in Proc. IEEE ICC'08, Beijing, China, May 2009.

[13] S. Shim, J. S. Kwak, R. W. Heath, and J. G. Andrews, "Block Diagonalization for Multi-User MIMO with Other-Cell Interference," IEEE Trans. Wireless Commun., vol. 7, no. 7, pp. 2671-2681, 2008.

[14] S. Ye and R. S. Blum, "Optimized Signaling for MIMO Interference Systems with Feedback," IEEE Trans. Signal Process., vol. 51, no. 11, pp. 2839-2848, May 2003.

[15] T. M. Cover and J. A. Thomas, Elements of Information Theory. NewYork, USA: ed. Wiley, 1991. 